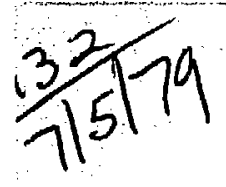

\title{
FHE ELECTRIC GUN: A NEW TOOL FOR ULTRAHIGH-PRESSURE RESEARCH
}

R. C. Weingart, H. H. Chau, D. R. Goosman,

W. W. Hofer, C. A. Honodel, R. S. Lee,

D. J. Steinberg, and J. R. Stroud

April 23, 1979

Work performed under the auspices of the U.S. Department of

Energy by the UCLLL under contract number W-7405-ENG-48.

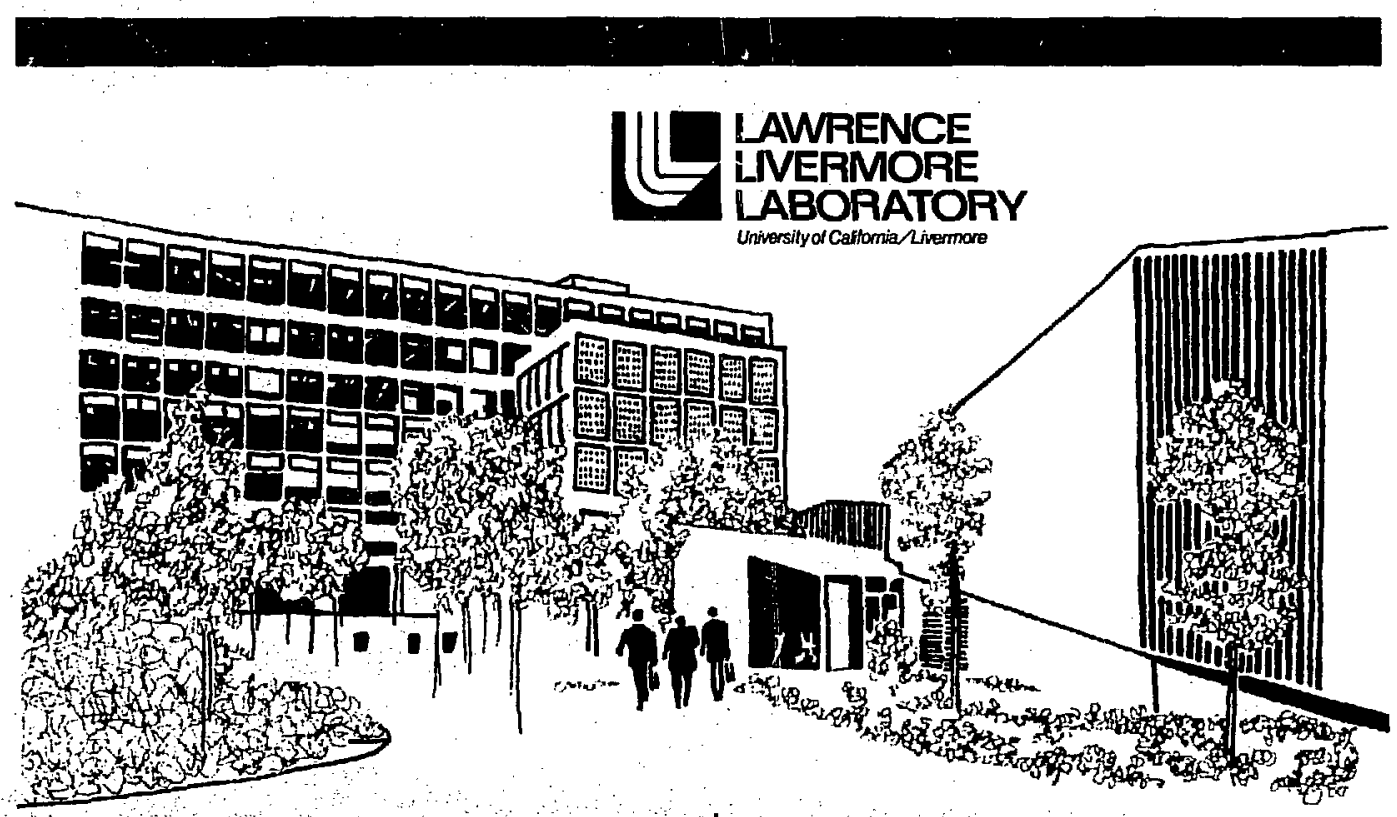




\section{近 \\ LAMRENCE LIVERMORE LABORATORY}

University of Cabfornia Livermore, Calitornia 94550

UCRL-52752

\section{THE ELECTRIC GUN: A NEW TOOL FOR \\ ULTRAHIGH-PRESSURE RESEARCH}

R. C. Weingart, H. H. Chau, D. R. Goosman,

W. W. Hofer, C. A. Honodel, R. S. Lee,

D. J. Steinberg, and J. R. Stroud

MS. date: April 23, 1979 


\section{CONTENTS}

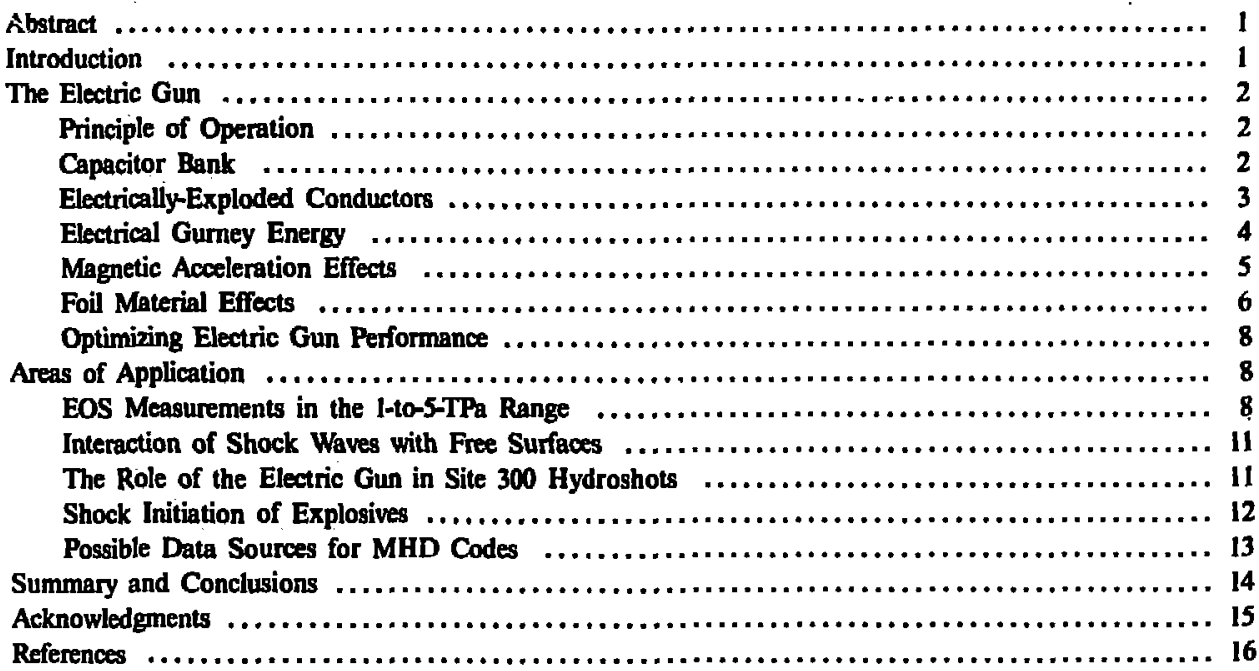

This report on petpered an an areant of work apartored by the United States Gomemmenc. Nelluter the Unlued Stater nor the United Stales Depulment of Enery. nor uny of thetr employets, ans any of theis contactorn, subcontracton, or thetr employee,s, muker any weinatify, exprets or inplied, of arymes any kal

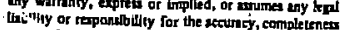
or une..' "רets of any informatlon, appentus, product or or une..' 'nets of any informetlon, appunitus, product or

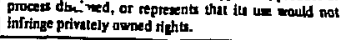




\title{
THE ELECTRIC GUN: A NEW TOOL FOR ULTRAHIGH-PRESSURE RESEARCH
}

\begin{abstract}
We have developed a new tool for ultrahigh-pressure research at LLL: This system, which we call the electric gun, has already achieved thin flyer plate velocities in excess of $20 \mathrm{~km} / \mathrm{s}$ and pressures of the order of 2 TPa in tantalum. We believe that the electric gun is competitive with laser- and nuclear-driven methods of producing shocks in the 1-to-5 TPa range because of its precision and ease and economy of operation. We encourage its development for shock initiation studies, dry runs for Site 300 hydroshots, and as a shock wave generator for surface studies.
\end{abstract}

\section{INTRODUCTION}

At a 1961 conference on exploding wires, papers were presented by Keller and Penning ${ }^{\prime}$ and Guenther et al. ${ }^{2}$ on the use of exploding metal foils to accelerate thin plates of dielectric material to velocities of 4-5 km/s. Keller's setup for obtaining fast plastic flyer plates, which he called "slappers", was used in the 1960's to study the effecis of shock waves on materials in the 0.001-to-0.01-TPa range. ${ }^{1-3}(1 \mathrm{TPa}=10 \mathrm{Mbar}$. In 1965, Stroud and Nikkola proposed using Keller's slapper system as the basis for a high explosive detonator. By 1976, Weingart et al. ${ }^{5}$ had extended the system's range to pressures of the order of 0.1.TPa and had used it to obtain quantitative data on short-pulse shock initiation of high explosives.

During the same period, we at LLL recognized the potential of the system as a tool for ultrahighpressure research (pressures $>0.5 \mathrm{TPa}$ ). Higher pressures were achieved by constructing composite flyers of dense metal and plastic ${ }^{6}$ Flyer velocity was increased by increasing electrical ciurrents so that magnetic forces became an important additional driving force on the flyer. Careful nyer laminate design and fast electrical current risetimes resulted in extremely planar flyer impacts.

To be consistent with terminology used to describe other precision shock wave generalors, we have named our flyer laminate and capacitor bank the electric gun. Our newest electric gun facility, designed specifically for equation-of-state (EOS) studies in the 1-to-5-TPa range, has already achieved thin flyer velocities in excess of $20 \mathrm{~km} / \mathrm{s}$. We believe the attainment of such velocities clearly places the electric gun in the front rank of ultrahigh-pressure shock wave generators.

Along with the ultrahigh-pressure capability come several experimental advantages:

- An electric gun facility is relatively inexpensive to run, benefitting programs that require a large number of experiments.

- The electric gun is small, simple, and can function onsite without the cumbersome operational procedures that are required when working with highexplosive generators, large lasers, or multi-stage gas guns. Close-in diagnostic access is available, experimental designs are not complicated by large expansion volumes, and turn-around time between experiments is short.

- The electric gun is a precision instrument. The flatness of the flyer impact compares favorably with that of projectiles from a two-stage light gas gun; the target is not preheated as in laser-driven shock waves; diagnostics are straightforward and available.

- Experiments can be done on small quantities of material in a disposable target chamber, permitting measurements on toxic materials that would otherwise require dedicated facilities.

In this report we will describe the electric gun facilities that are operational at LLL and discuss applications of electric gun technology to problems of interest to shock wave researchers at LLL and other laboratories. 


\section{THE ELECTRIC GUN}

\section{PRINCIPLE OF OPERATION}

The operation of the electric gun is based on exploding a thin metallic foil by discharging a capacitor bank connected to the foil. If the discharge time of the bank is short compared to thermal diffusion times away from the foil, ohmic heating can deposit a large amount of energy into the foil. The deposited energy is released in the subsequent expansion of the foil material, which acts as a driver gas to accelerate thin plates positioned adjacent to the foil. If large currents are employed to explode the foil, sizeable magnetic forces arise; these also accelerate the flyer plate.

Figure 1 illustrates the design of the assembly we use to couple the energy of the exploding metal foil to a thin flyer plate. A flat-conductor transmission line delivers current from the capacitor bank into a foil, which bridges a gap in one of the heavier conductors. The foil is designed to be the most resistive element in the circuit; it absorbs most of the energy delivered by the bank (until after foil explosion, when a low resistance current sheet is formed in its place). The flyer-plate material is laid on top of the foil and the transmission line, foil, and flyer-plate material are thermally bonded into a stiff assembly that is easy to handle and connect to the capacitor bank. The explosion of the foil and the accompanying magnetic forces punch out a section of the flyer-plate material and accelerate it up a few-millimeter-long barrel to strike a target.

\section{CAPACITOR BANKK}

The electric gun is connected to a series RLC circuit as illustrated in Fig. 2. $\mathbf{R}_{\mathrm{g}}$ and $\mathrm{L}$ represent the distributed resistance and inductance of the capacitor bank, transmission line, and switch. To achieve maximum current output, the bank is operated in an underdamped mode. To define the important parameters of the bank we consider the current, I, delivered

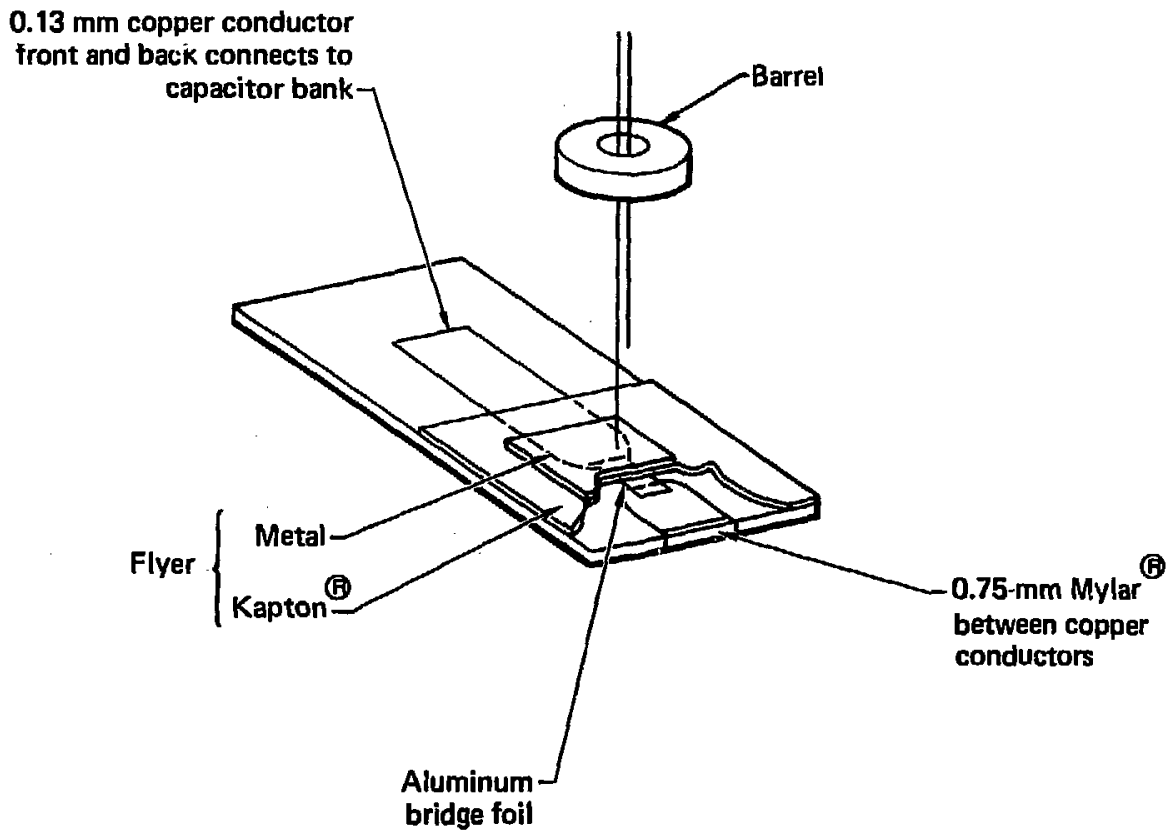

Fig. 1. Assembly design for coupling the energy of the exploding metal foil to a thin flyer plate. 


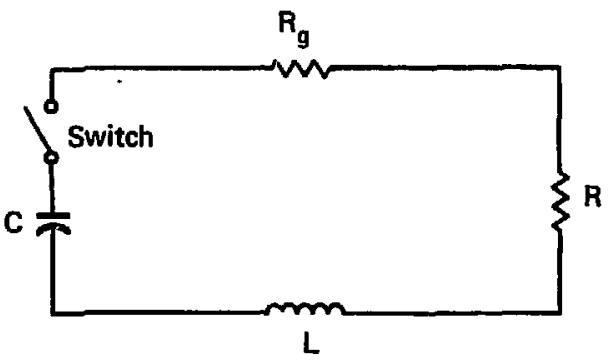

Fig. 2. Diagram of the series RLC circuit to which the electric gun is connected. $\mathbf{R}_{\mathbf{g}}$ and $L$ are the distributed resistance and inductence, respectively, of the capacitor bank (C), transmission line, and switch of the circuit.

into a static load of resistance $R=\mathbf{R}_{\mathbf{g}}$ when the bank is changed to a voltage $V_{0}$. $I$ is given by:

$$
I=I_{m} \sin (\omega t) \exp (-t / \tau)
$$

where:

$I_{m}=V_{0} / L \omega, \tau=2 L / R$, and $\omega=\left(\frac{1}{L C}-\frac{R^{2}}{4 L^{2}}\right)^{1 / 2}$.

We presently use several different capacitor banks, depending on the application. One bank, which we have used extensively in high explosive initiation studies, has a capacitance of $56 \mu \mathrm{F}$, a system inductance (bank plus load) of $40 \mathrm{nH}$, and a peak charging voltage of $40 \mathrm{kV}$. For $22 \mathrm{~m} \Omega$ circuit resistance, $\omega=6.1 \times 10^{5} \mathrm{~s}^{-1}$, $I_{m}=1.64 \mathrm{Ma}$, and $\tau=3.6 \times 10^{-6} \mathrm{~s}$. Another important bank parameter is the initial rate of current rise, which is given by

$$
\left.\frac{d I}{d t}\right)_{t=0}=\frac{V_{0}}{L}
$$

For the bank described above, $(\mathrm{dI} / \mathrm{dt})_{t=0}=1 \mathrm{TA} / \mathrm{s}$.

We have recently acquired a new bank that is significantly faster. In its present configuration, the fast bank has $17.2 \mu \mathrm{F}$ capacitance, $16 \mathrm{nH}$ system inductance and a peak charging voltage of $100 \mathrm{kV}$. For $22 \mathrm{~m} \Omega$ resistance these parameters lead to the values $\omega=1.5 \times 10^{6} \mathrm{sec}^{-1}, I_{\mathrm{m}}=4.0 \mathrm{MA}, \tau=2.2 \times 10^{-6} \mathrm{~s}$, and $\mathrm{dI} / \mathrm{dt})_{t=0}=6.2 \mathrm{TA} / \mathrm{s}$. By optimizing insulation thickness to reduce inductance and by using a higher charging voltage, we hope to achieve even faster risetimes and higher peak currents.

\section{ELECTRICALLY-EXPLODED CONDUCTORS}

A large body of work on exploding conductors exists in the literature. ${ }^{7-10}$ We will briefly review some important features, making simple assumptions that allow a qualitative picture of exploding foil behavior.

Consider a conductor of resistance $\mathbf{R}$ that carries a current $I(t)$. If we assume that no heat escapes from the conductor, the rate of change of conductor temperature is

$$
\frac{d\left(T-T_{0}\right)}{d t}=\frac{I^{2}(t) R}{M c}
$$

where $T_{0}$ is the initial temperature, $\mathrm{c} i$ i the specific heat (assumed constant) and $M$ is the rnass of the conductor. If we further assume that $R$ is a linear function of temperature given by

$$
\mathbf{R}=\mathbf{R}_{\mathbf{0}}\left[\mathbf{1}+\left(\mathrm{T}-\mathrm{T}_{0}\right)\right] \text {, }
$$

where $\mathbf{R}_{\mathbf{0}}$ is the resistance at temperature $T_{0}$ and $\alpha$ is the temperature coefficient of resistivity, then, as Bennett ${ }^{8}$ has shown,

$$
T-T_{0} \simeq \frac{1}{\alpha} \exp \left[\frac{R_{0} \alpha}{M c} \int_{0}^{t} I^{2}(\lambda) \mathrm{d} \lambda\right] .
$$

Equation (5) predicts a very steep temperature rise as current continues to flow through the conductor. At some limiting temperature, the thermal pressure in the conductor becomes so great that an explosion occurs. Even though Eq. (5) is based on rather unrealistic assumptions about the temperature dependence of the resistivity and the specific heat, it gives a qualitative picture of very steep rise in temperature that occurs just before burst. Logan et al. "1 used a similar model to compute temperature distributions in exploding foils and were able to successfully compute burst times and burst currents for several different foil materials and sizes.

It is found experimentally that the argument of the exponential term in Eq. (5) plays a crucial role in exploding conductor phenomena. The argument can be rewritten as

$$
\frac{\alpha}{\sigma_{0} \rho_{0} \mathrm{c}} \int_{0}^{t} \mathrm{~J}^{2}(\lambda) \mathrm{d} \lambda
$$


where $\sigma_{0}$ is the initial conductivity, $\rho_{0}$ the initial density, and $J$ the current density. The quantity

$$
g=\int_{0}^{l} B J^{2}(\lambda) d \lambda
$$

is called the specific action, where $t_{B}$ is the time between the current start and the bursting of the conductor. Experiments have shown that, for conductors with a round cross section, the specific action is independent of wire dimensions and current wave form $^{10,12}$; hoivever, for foils, the specific action varies significantly with foil geometry and current waveform. $^{11,13}$ Even so, the assumption of a constant specific action to burst is useful for making parameter studies.

\section{ELECTRICAL GURNEY ENERGY}

The velocity that can be imparted to a thin flyer plate placed on top of an exploding foil depends on the foil material, foil mass, flyer-plate mass, and the current waveform. An electrical analog of the Gumey model, proposed by Tucker and Stanton, ${ }^{14}$ has proved extremely useful in making parameter studies on exploding-foil systems. The Gurney theory, ${ }^{15}$ originally developed to predict velocities of fragments accelerated by high-explosive charges, predicts plate velocities from a characteristic energy, $E_{g}$, which is related to the maximum velocity an explosive charge is capable of delivering to a plate. A linear velocity profile is assumed in the explosive reaction products, and kinetic energy is partitioned between the plate and the reaction products so as to conserve momentum. For a onedimensional system, where an explosive sheet of mass $C$ per unit area is backed by an infinite mass on one side and a plate of mass $M$ per unit area on the other, the final plate velocity is:

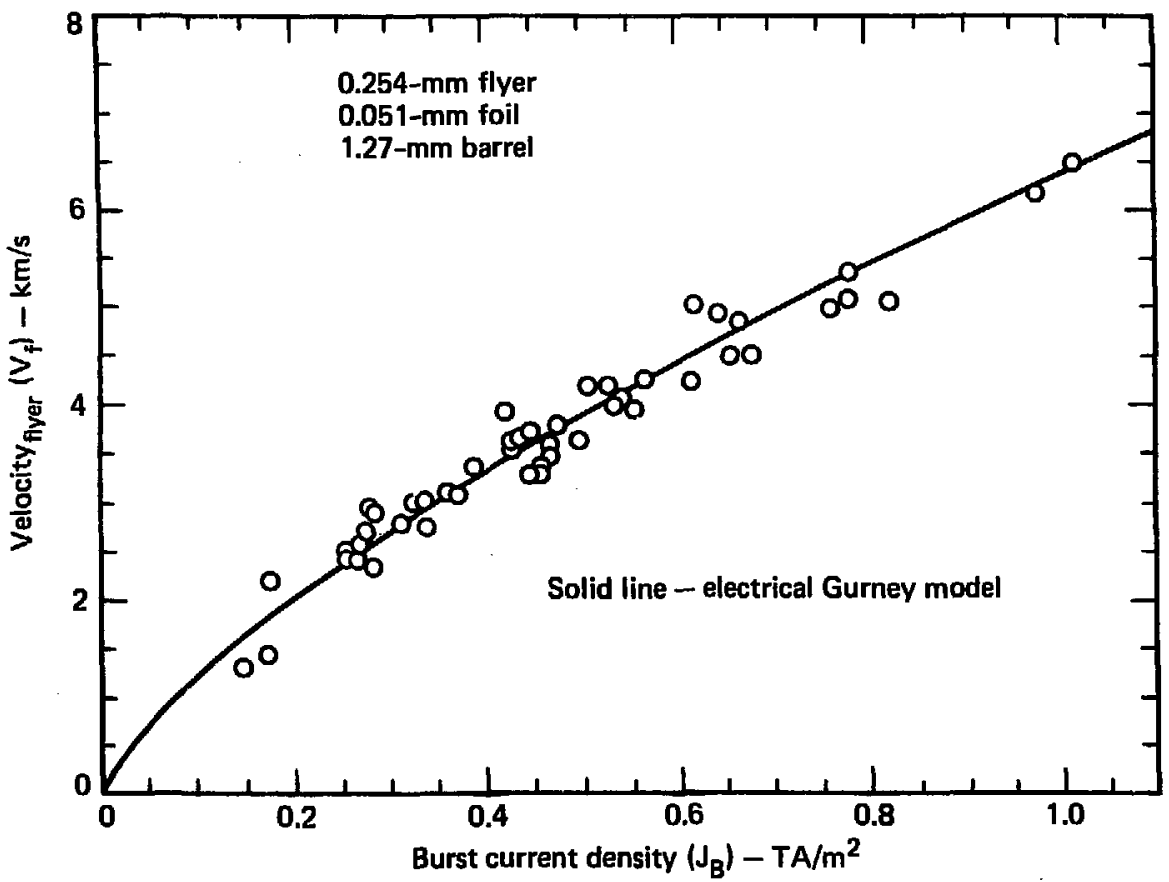

Fig. 3. Comparison of experimental flyer data and predictions of the electrical Gurney model. The experiment was run with an aluminum foil and the slower capacitor bank; barrel length and $M / C$ were held constant. 
Tucker and Stanton assumed that the characteristic energy associated with an electrically-exploded foil depended only on the burst current density of the foil, $\mathbf{J}_{\mathbf{B}}$. They wrote the Gurney equation in the form

$$
\begin{aligned}
V_{\mathrm{I}} & =\sqrt{K J_{B}^{b}}\left(\frac{M}{C}+1 / 3^{-1 / 2}\right) \\
& =V_{G}\left(\frac{M}{C}+1 / 3\right)^{-1 / 2}
\end{aligned}
$$

where $K$ and $b$ are empirically-determined constants and $V_{G}$ is a characteristic velocity we will call the Gurney velocity. The constant $K$ depends on the barrel length, so Eq. (9) can be applied only to one barrel length for a particular choice of $K$. The constant $b$ is approximately independent of barrel lengths in the 1-to-5-mm range. Figure 3 shows an electrical-Gurneymodel fit to some of our flyer data, where the slower bank described above was used as the energy source and the foil material was aluminum. The data of Fig. 3 are for a constant barrel length and a constant ratio of explosive mass to plate mass, $M / C$, but we have found that the Gurney model represents the data over a wide range of values of $M / C$, foil thickness, and width.

The electrical Gurney energy for the data of Fig. 3 depends on burst current density according to the relation

$$
\sqrt{2 E_{g}}=15.6 \mathrm{~J}_{\mathrm{B}}^{0.768}
$$

where the burst current density, $J_{B}$, is expressed in $T A / m^{2}$ and $\sqrt{2 E_{g}}$ is in $\mathrm{km} / \mathrm{s}$. With the slower bank, the maximum $J_{B}$ is about $1.1 \mathrm{TA} / \mathrm{m}^{2}$, resulting in $\sqrt{2 E_{\mathrm{g}}}=16.8 \mathrm{~km} / \mathrm{s}$. The primary reason for our acquisition of the fast bank was the hope that we could increase $J_{B}$ by a factor of about 4 , and achieve thin flyer velocities in the $30-\mathrm{km} / \mathrm{s}$ range.

It is interesting to compare the Gurney energy of electrically-exploded foils with the Gurney energy of HMX, a powerful high explosive. Equation (8) predicts a limiting plate velocity of $\sqrt{6 \mathrm{E}_{\mathrm{g}}}$ as $\mathrm{C}$ becomes large, so we will compare values of $\sqrt{2 E_{g}}$. For $\mathrm{HMX}$, $\sqrt{2 \mathrm{E}_{\mathrm{g}}}=2.97 \mathrm{~km} / \mathrm{s}^{15}$, while for the electrically-exploded foil data shown in Fig. 3, values of $\sqrt{2 \mathrm{E}_{\mathrm{g}}}$ range up to $14 \mathrm{~km} / \mathrm{s}$. The ultimate velocity that an explosive can impart to a plate is determined by the thermal energy and EOS of the detonation products. The extremely large Gurney energies we observe in electrically exploded foils can be attributed, in part, to the large thermal energies that can be electrically deposited in a foil. For example, a: $\mathbf{4 0} \mathrm{kV}$, our slower bank is capable of depositing - up to the time of burst approximately $3 \mathrm{~kJ}$ of eriergy in a 2.54- $\times 2.54-$ $\times 0.0051-\mathrm{cm}$ aluminum foil. The cohesive energy of aluminum is $11.9 \mathrm{MJ} / \mathrm{kg}^{16}$; subtracting this from the deposited energy density leaves $22 \mathrm{MJ} / \mathrm{kg}$ excess thermal energy in the foil at burst.

The heat of detonation of HMX is about $6 \mathrm{MJ} / \mathrm{kg}^{17}$ So it appears that the specific energy available for plate acceleration is greater for electrically exploded foils than for HMX. It is not meaningful, however, to directly compare electrical Gurney energy with HMX Gurney energy for high current systems without correcting for magnetic acceleration effects.

\section{MAGNETIC ACCELERATION EFFECTS}

The dependence of electrical Gurney energy on barrel length is an interesting phenomenon. Kury et al. ${ }^{18}$ have reported that, over a range of common values of $\mathbf{M} / \mathbf{C}$, detonation of a chemical explosive drives a plate to nearly its final velocity by the time the detonation products have expanded to twice their initial volume. This is contrary to our experiments with electrically-exploded foils, where we observe significant acceleration of thin plates after a 100-fold expansion of the foil. ${ }^{5}$ This continued acceleration occurs for two reasons. One reason is that even though energy deposition is very sharply peaked near burst time, some energy continues to be deposited into the foil material after burst. The primary reason, however, for the continued acceleration after burst is the magnetic pressure developed between the foil and the return conductor of the transmission line. VISAR laser interferometer measurements have confirmed the presence of this magnetic acceleration. Figure 4 shows VISAR velocity-time records from three experiments that were specifically designed to study magnetic acceleration. Three laminates were prepared that used foils with the same cross-sectional area but different thicknesses. They all burst at roughly the same burst current density and experienced the same current waveform, but the current per unit width varied by a factor of four. A Gurney model is inappropriate to describe the data of Fig. 4a because the Gurney model assumes that the plates have reached their final velocity, while the plates are clearly being accelerated throughout the duration of the experiment. A thorough 
mathematical description of the plate motion would require a magnetohydrodynamic (MHD) calculation coupled to a calculation of the electrical energy deposited into the foil material as it expands. We have not yet attempted such a calculation, but will try to qualitatively account for the observed motion by separating the magnetic and hydrodynamic effects.

The magnetic contribution to the velocity was estimated by assuming infinite plane conductors and integrating the equation

$$
\left(\rho_{c} d_{c}+\rho_{m} d_{m}\right) \ddot{x}=\frac{\mu_{0} \lambda^{2}}{2}
$$

where $p_{c}, d_{c}$, and $p_{m}, d_{m}$ are the density and thickness of the foil and flyer material, respectively, $\lambda$ is the current per unit width calculated from Equation (1); $\mu_{0}$ is the permittivity of free space; and $\ddot{X}$ is the acceleration of the foil and flyer. If the magnetic and hydrodynamic contributions to the flyer velocity are separable, subtracting the magnetic contribution from a measured vclocity-time curve should leave the hydrodynamic contribution. Figure $4 b$ shows the curves of Fig. $4 \mathrm{a}$, with the magnetic contributions subtracted. It is, at first glance, surprising that the curves of Fig. $4 b$ lie so close together because the mass/area of vaporized foil material driving the flyer differs by a factor of four for the three curves. However, if we look again at Fig. $4 a$, we see that the thicker foil has moved appreciably before burst, giving the vaporized foil a larger initial volume and a lower initial pushing pressure. Siegel, in his monograph on high-speed gurs, ${ }^{19}$. shows that the final velocity of a projectile accelerated by a driver gas depends on the ratio of the initial pressure to the mass of the driver gas. Thus, it is not unreasonable that the thinnest foil provided the same hydrodynamic push as the thickest.

Since magnetic pressure increases as the square of $\lambda$, magnetic forces will be particularly significant for the faster capacitor bank system. Preliminary experiments with the faster system have indicated serious discrepancies between the electrical Gurney theory and the observed flyer velocities. It appears that under conditions of very large $\lambda$ the Gurney theory is no longer directly applicable and magnetic effects must be explicitly considered.

\section{FOIL MATERIAL EFFECTS}

We have conducted tests on thirteen different materials to ascertain whether there is an optimum material to use as the exploding foil. We will first compare Gurney energies. For most of the materials

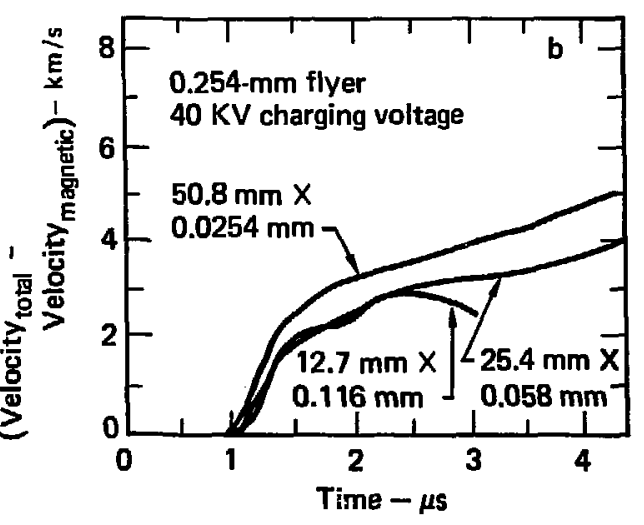

(b)

Fig: 4: VISAR laser interferometer confirmation of the presence of magnetic acceleration in 3 laminates. The foils in each laminate had the same cross-sectional area but the thicknesses differed as shown. Each lominate burst around $0.5 \mathrm{TA} / \mathrm{m}^{2}$ burst current density and experienced the same current waveform. However, the currents per uuit width varied by a factor of 4. Fig. 4a) Velocity-time records for the 3 laminates. Fig. (b) The curves recorded in $4 a$, with the magnetic contribution subtracted. Note that hydrodynamic push is independent of laminate thickness. 
we do not yet have sufficient experimental data to compute $K$ and $b$ from Eq. (9). Since the burst currents are not the same, we need some way of normalizing the results to the same $J_{B}$ so that the magnetic contribution will be constant. We will assume that $b$ is the same for all materials and compute $a$ normalized Gurney velocity,

$$
V_{N}=\sqrt{2 E_{g}}\left(\frac{0.5}{J_{B}}\right)^{b / 2},
$$

where the value of $b$ has been determined from extensive experiments on aluminum foils to be $1.54, \sqrt{2 \mathrm{E}_{\mathrm{g}}}$ is determined from the measured flyer velocity, foil mass/area and flyer mass/area, and the burst current density is normalized to a value of $0.5 \mathrm{TA} / \mathrm{m}^{2}$. Thi: different materials, $M / C, \sqrt{2 E_{g}}, J_{B}$ and $V_{N}$ are listed in Table 1.

For a $\gamma$-law gas the speed of sound is:

$$
c^{2}=\gamma \mathrm{PV}=\frac{\gamma \mathrm{RT}}{\mathrm{m}},
$$

where $c$ is the speed of sound, $\gamma$ the ratio of specific heats, $R$ the gas constant and $m$ the molecular weight. Because a driver gas must be able to communicate with the piston it is pushing, the speed of sound limits the attainable piston velocity. Equation (13) predicts a maximum flyer velocity proportional to $\mathrm{m}^{-\mathrm{t} / 2}$.
Such a dependence is consistent with the data of Table 1 , as we show in Fig. 5 , where $V_{G}$ is plotted as a function of $M$. The solid curve was determined by fitting a linear regression model to the data. We belicve the large scatter in the data is due to the very limited number of shots we expended in investigating foil material effects. The asymptotic value of velocity in Fig. $4 b$, as $m$ continues to increase, may be due to the magnetic contribution to the velocity.

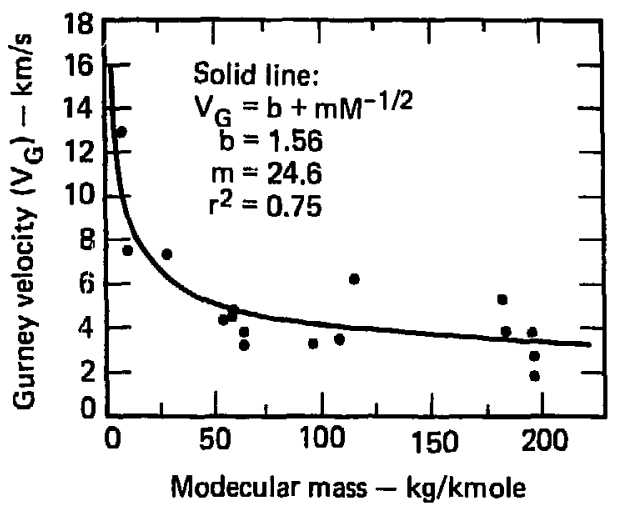

Fig. 5. $V_{G}$ plotted as a function of molecular mass, $m$. The solid line is a linear regression curve fitted to the experimental data. The wide scatter is likely an artifact of the small sample size.

TABLE 1. Summary of performance of different foil materials. $J_{B}$ is burst current density, $M$ is flyer mass/ area, $C$ is foil mass/area, $V_{f}$ is the measured flyer velocity at the listed burst current density, $2 E_{g}$ is the Gurney velocity and $V_{N}$ is the normalized Gurney velocity.

\begin{tabular}{lccccc}
\hline Material & $\begin{array}{c}J_{\mathbf{B}} \\
\left(\mathbf{T A} / \mathbf{m}^{2}\right)\end{array}$ & $\mathbf{M} / \mathbf{C}$ & $\begin{array}{c}\mathbf{V}_{\mathbf{f}} \\
(\mathbf{k m} / \mathbf{s})\end{array}$ & $\begin{array}{c}2 E_{\mathbf{g}} \\
(\mathbf{k m} / \mathbf{s})\end{array}$ & $\begin{array}{c}\mathbf{V}_{\mathbf{N}} \\
(\mathbf{k m} / \mathbf{s})\end{array}$ \\
\hline Alurninum & 0.54 & 1.3 & 6.1 & 7.8 & 7.4 \\
Beryllium & 0.40 & 3.8 & 3.1 & 6.3 & 7.5 \\
Copper & 0.72 & 0.4 & 6.0 & 5.1 & 3.9 \\
& 0.93 & 0.8 & 5.0 & 5.3 & 3.3 \\
Gold & 0.49 & 0.2 & 3.8 & 2.7 & 2.7 \\
Indium & 0.69 & 0.4 & 3.0 & 2.5 & 2.0 \\
Lithium" & 0.23 & 0.2 & 4.7 & 3.4 & 6.2 \\
Molybdenum & 0.32 & 8.8 & 3.0 & 9.1 & 12.8 \\
Niekel & 0.35 & 0.2 & 3.5 & 2.5 & 3.3 \\
& 0.70 & 1.6 & 4.4 & 6.0 & 4.6 \\
Platinum & 0.52 & 0.8 & 4.6 & 4.9 & 4.8 \\
Silver & 0.28 & 0.1 & 3.8 & 2.4 & 3.8 \\
Steel & 0.62 & 0.3 & 5.0 & 4.1 & 3.5 \\
Tantalum & 0.48 & 0.4 & 5.0 & 4.4 & 4.5 \\
Tungsten & 0.26 & 0.1 & 5.1 & 3.2 & 5.3 \\
\hline
\end{tabular}

\footnotetext{
a Barmel length was $4 \mathrm{~mm}$.
} 


\section{OPTIMIZING ELECTRIC GUN PERFORMANCE}

The interactions among the experimental parameters of the electric gun make the task of optimization very complex. In some cases, the interactions are poorly understood and we have no alternative to an empirical approach. We will describe some of the interactions we understand and point out areas where our basic understanding is lacking. The electrical characteristics of the capacitor bank are the starting point for our discussion, since bank performance will dictate many of the other parameters. To accelerate a flyer plate that remains flat as it moves down the barrel, it is important to assure that the foil bursts within a $\mu$ s or less from current start. If this requirement is not met, magnetic pressure will overcome the inertia of the foil and flyer, producing a distortion in the foil geometry before burst occurs; if this occurs, the flyer impact will not be as flat. The burst time requirement can be met in two ways. Choosing a capacitor bank with a minimal inductance and maximum practical charging voltage allows the greatest experimental flexibility. Since the rate of current increase is proportional to the ratio of charging voltage to inductance, this will assure minimum burst times. Once the capacitor bank parameters are fixed, the experimenter can still vary the burst time by varying the foil cross. sectional area. Because the specific action to burst is roughly constant for a given material, Eq. (7) defines an approximate relationship between burst time and cross-sectional area of the foil.

In addition to the requirement of planar plate impact, shock wave experiments require a pressure pulse of known amplitude and of sufficient duration to make precise measurements. The amplitude of the pulse is controlled by the speed and shock impedance of the flyer plate material. The material adjacent to the foil must be a dielectric (we have found films of
Kapton ${ }^{\oplus}$ polyimide and Mylar ${ }^{\oplus}$ polyester most suitable*), but a metal foil may be placed on top of the dielectric layer if higher pressures are desired. The dependence of flyer velocity on burst current density, flyer mass/area and foil mass/area is described by Eq. (9).

For a particular foil cross-sectional geometry, the choice of foil material will determine the characteristic velocity $V_{G}$ and the foil mass/area, $C$. Inspection of the data of Fig. 5 might lead one to believe that foils with the largest normalized $V_{G}$ are the most desirable, but this is not the case. The materials with the largest VG are, in general, those with the lowest density. Since the flyer velocity depends on $M / C$ as well as $V_{G}$, a thicker foil must be used for the lower density materials to achieve the same value of $\mathrm{M} / \mathrm{C}$ as for a denser material. The capacitor bank parameters, however. limit the thickness of the foil if the requirement of burst within a microsecond or less is to be met. The dependence on $\mathbf{M} / \mathbf{C}$ may be less, however, if magnetic effects are important. In this case, a lower-density foil material might be desirable. With very fast-rising currents, or thick foils, or both, magnetic diffusion will also play a role. Another material property that limits the performance of a potential candidate material is the specific action to burst. Some of the higher density materials showed reasonable values of $V_{G}$ but had rather large specific actions, leading to long burst times and poor-quality flyers. We are by no means certain that we have found the optimum foil material, but our experience to date has shown aluminum to be the material of choice, and most of our electric gun work over the last few years has been with aluminum foils. In light of our imperfect understanding of the effects discussed above, the availability of a much faster bank suggests that a re-examination of some of the previously-studied materials would be worthwhile.

\section{AREAS OF APPLICATION}

\section{EQUATION OF STATE MEASUREMENTS IN THE 1-TO-5-TPa RANGE}

Present experimental techniques allow us to reach pressures of approximately $1 \mathrm{TPa}$, and theory is believed reliable above approximately $10 \mathrm{TPa}$. Equations of state have relied on interpolation schemes to fill in the intermediate range. Our electric gun gives promise that we may be able to experimentally investigate this intermediate region.

The experimental arrangement for an EOS measurement is shown in Fig. 6. The flyer is a Kapton ${ }^{\oplus}$ metal laminate that accelerates down a few-millimeterlong barrel to strike the target. The thin metal layer placed on top of the Kapton ${ }^{\varpi}$ flyer increases the total

-Kapton and Mylar are trademarks for E.I. du Pont de Nemours \& Company's polyimide aromatic and polyester film, respectively. 


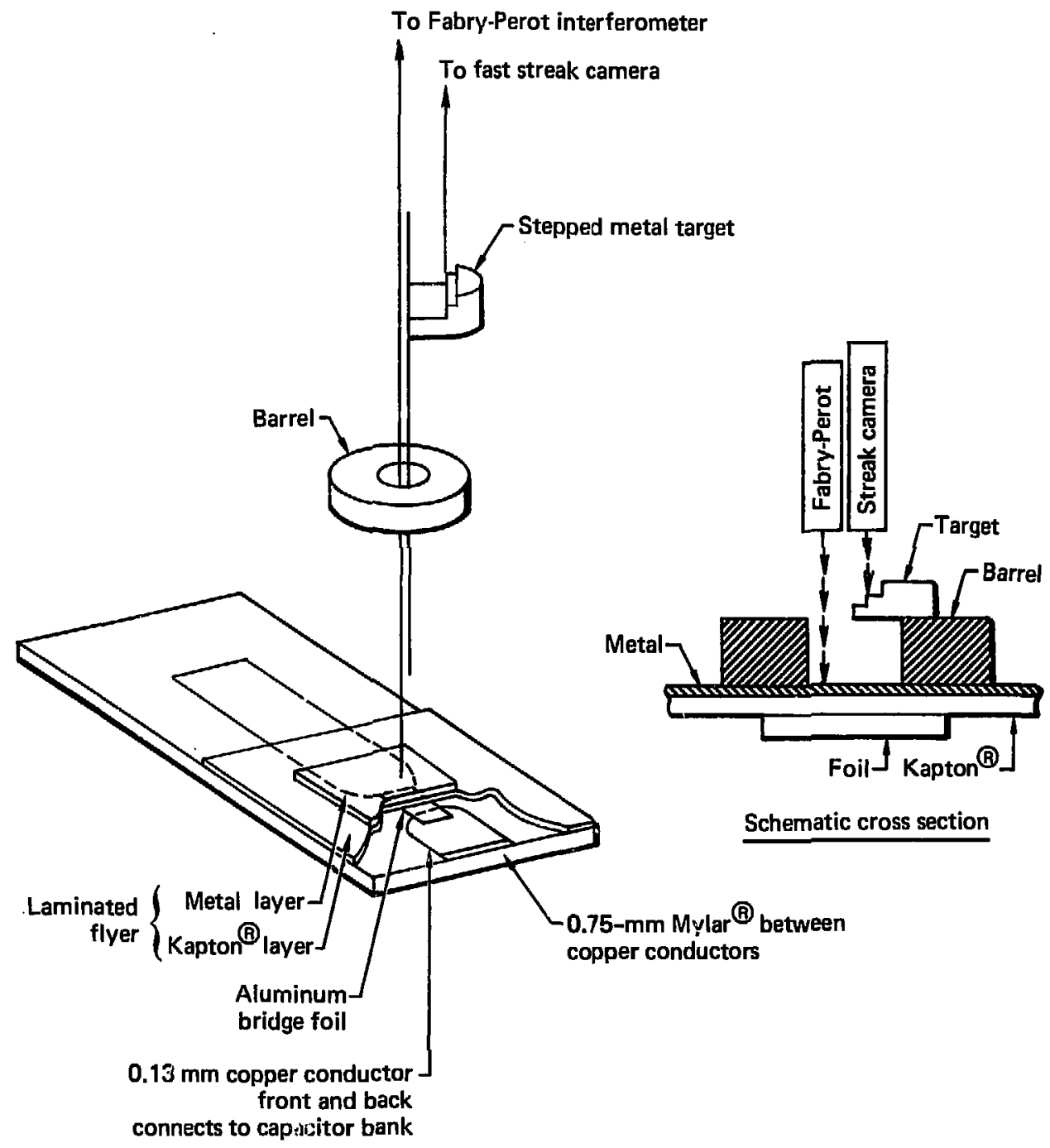

Fig. 6. The experimental arrangement for an EOS measurement. The metal fyer is typically a few tens of microns thick, and the target is approximately 2 to 3 times the thickness of the fyer.

mass of the flyer and therefore lowers its velocity. However, the shock impedance or "stiffness" (the product of the density and the shock velocity) is so much greater for a typical metal than for Kapton ${ }^{\otimes}$, that even at the lower velocity the metal flyer induces a much greater pressure in the target. The target is the same metal as the flyer and has a set of precisely cut steps in it. A Fabry-Perot interferometer continuously monitors the velocity of the flyer.

At the time of fyer-target impact, symmetry considerations show that the material velocity, $U_{p}$, in the shock target is exactly equal to one-half the flyer velocity. At pressures $\geq 0.1 \mathrm{TPa}$, shock heating of the material produces thermal light emission sufficient to be detected by a fast streak camera at the instant the shock arrives at the free surface.* Measuring shock velocity, $U_{5}$, then consists of measuring

*Actually, our present camera sees thermal emission from the unloaded hot target. Its time resolution is not adequate to see the shock at the instant of emergence from the surface. 
shock-transit time across the steps in the free surface of the target. Using the measurements of $U_{s}$ and $U_{p}$, and the mass and momentum conservation conditions across the shock front, we can determine the pressure and volume on the Hugoniot. Measurements are performed in a vacuum to eliminate effects of the air that otherwise would be compressed between the flyer and target.

The electric gun is competitive with laser- and nuclear-driven experiments in producing shocks in the 1-to-5-TPa range. To measure a point on a Hugoniot, two independent measurements must be made, e.g., $U_{s}$ and $U_{p}$. The technique for measuring $\mathrm{U}_{p}$ in a nuclear-driven experiment is complicated and has a precision of only $\pm 5 \%$. $^{20}$ The laser system does not yet have a proven method of measuring $U_{p}$, so presently it must rely on theoretical calculations to supply the missing information. ${ }^{21,22}$ However, with the electric gun, we can use a precise method of determining $U_{p}$, i.e., using a symmetric impact and measuring the flyer velocity. Finally, the low cost and relative ease of doing many laboratory experiments, even in a glove box, make the electric gun especially attractive.

Figure 7a shows a streak record made when a tantalum flyer impacted a tantalum target that had a step cut in the middle of the free surface. The measured shock velocity is $6.1 \mathrm{~km} / \mathrm{s} \pm 10 \%$. Using a flyer velocity of $5.2 \mathrm{~km} / \mathrm{s}$, estimated from previous

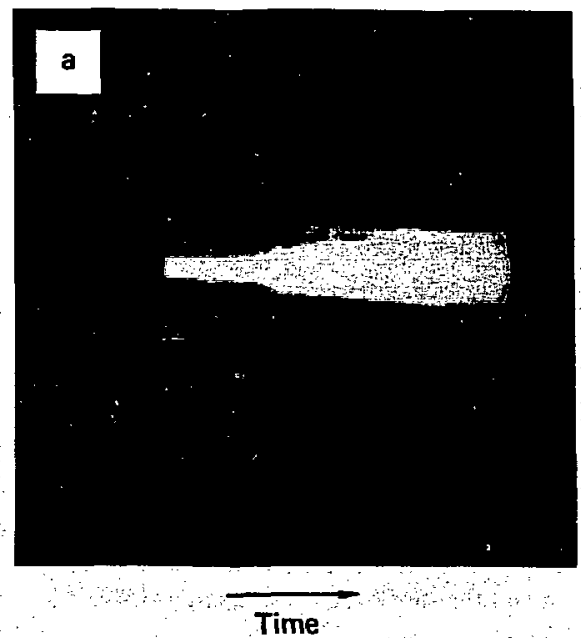

experiments, the shock pressure is approximately $0.3 \mathrm{TPa}$. The shock velocity at this pressure compares favorably with published data. The pressure produced in this preliminary experiment is approximately equal to the maximum pressure that can be produced in tantalum with the fastest two-stage gas-guns.

Figure $7 b$ shows the result of a similar experiment using a flat target. The simultaneity of the start of thermal emission demonstrates the flatness or "onedimensionality" of the gun over a diameter of approximately $3 \mathrm{~mm}$. Flyer flatness at impact has been estimated as better than $8 \mu \mathrm{m}$; and the turn-on of the light emission from the target is less than 600 psec.

Recently, we measured a velocity of approximately $16 \mathrm{~km} / \mathrm{s}$ for a $12.7-\mu \mathrm{m}$-thick, 5.08 -mm-diameter, composite tantalum/plastic flyer. Had this flyer impacted a tantalum target, a shock of nearly 2 TPa would have been produced. Recent experiments with the fast bank have accelerated Kanton flyers to velocities in excess of $20 \mathrm{~km} / \mathrm{s}$. At $20 \mathrm{~km} / \mathrm{s}$ a symmetric impact between a tungsten projectile and target will generate $\sim 3 \mathrm{TPa}$.

As can be easily seen in Fig. 7a, the present streak camera is too slow to be useful in Hugoniot experiments. We are presently installing a new camera with a maximum writing speed that is approximately 100 times faster. This will allow us to spread out our streak records in time and make shock-velocity measurements with greater precision. Our immediate objective is to simultancously ineasure $U_{p}$ and $U_{s}$ at several

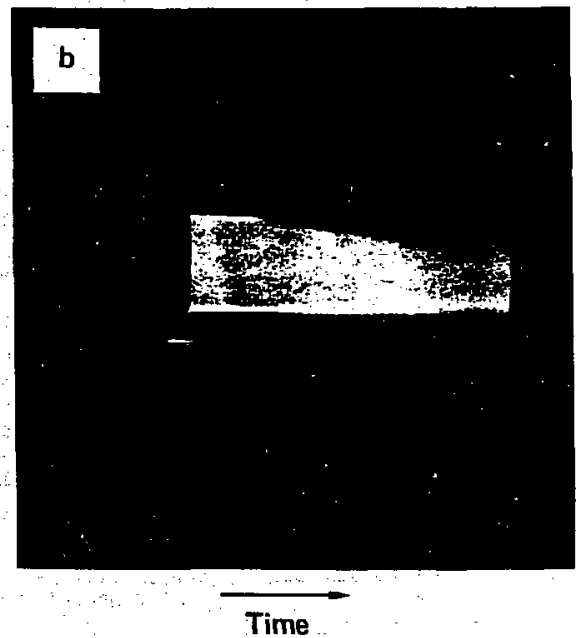

Fig. 7a. Streak-camera record of the start of luminescence of a stepped (step cut in the middle of the surface) tantalum terget. Fig. Th. Streak-camera record of the start of luminescence of a flat tantalum target. Both targets shocked to $\sim 0.3$ TPa: 
pressures between approximately 0.1 and $0.5 \mathrm{TPa}$ in a material with a well-characterized Hugoniot, such as tantalum or platinum.

\section{INTERACTION OF SHOCK WAVES WITH FREE SURFACES}

Observation of the response of the free surface of a solid body to the passage of a shock wave is a common method for studying material behavior at high pressures. The observed property of the free surface may be its velocity, brightness, reflectivity, or general appearance. Common measuring instruments are laser velocimeters, contactor pins, pyrometers, high-speed cameras, pressure gauges, and short pulse $\mathrm{X}$-ray generators. Free-surface measurements are essential in evaluating the performance of HE driven plates and are used to probe basic material properties. Although various sorts of free-surface measurements have been made for many years, the response of free surfaces to shock waves is poorly understood. In the past, many phenomena such as changes in reflectivity or color of shocked surfaces have been regarded as interesting or annoying artifacts but not as objects for study. We can no longer afford to ignore these effects, because to fully exploit the capabilities of advanced diagnostics, e.g. the laser velocimeters, we must learn how to predict and control phenomena like reflectivity changes in shocked surfaces.

Ejection of particulate material from shocked surfaces is a long-standing experimental problem that demands systematic study. The origin of surface ejecta is unclear and may be due to microjetting, hydrodynamic instabilities, melting, or foreign material. If the shocked surface is in close proximity to a thermonuclear plasma, ejection of foreign material into the plasma can cause serious energy losses from the plasma.

Knowledge of instantaneous material temperatures is fundamental to accurate modeling of shockheated solids. Radiation pyrometry, though limited to free surfaces and transparent materials, can be used to make dynamic temperature measurernents with very short response time over a wide temperature range. Radiation pyrometers measure the radiance of the heated sample over one or more wavelength bands. Planck's law can then be used to calculate the temperature if the radiation distribution approaches that of a blackbody radiator. If two or more wavelength bands are used, geometrical factors may be cancelled and the ratio of the band emittances used to calculate the temperature.
With ultrafast pyrometers we can measure shock temperatures directly by observing radiation from the shock wave shortly before it reaches the free surface of the material. A temperature measurement of the shock compressed material followed by a similar measurement on the unloaded material will help guide our EOS modeling. Additionally, the fast time response system will help us identify the source of surface instabilities. The pursuit of this challenging work will fill many gaps in the understanding of shockheated solids.

The electric gun is an ideal shock wave generator for the surface studies described above. It can produce well-defined pressure pulses in any pressure range of practical interest. The duration of the pulse, while short compared to gun or explosively-driven shocks, is more than adequate for the study of phenomena that occur directly on a shocked surface. Perhaps most important, the convenience and low cost of the electric gun allow a large number of experiments at a reasonable cost in both dollars and manpower.

\section{THE ROLE OF THE ELECTRIC GUN IN SITE 300 HYDROSHOTS}

The diagnostic technique of laser velocity interferometry, only recently successful in observing the velocity time history of plates accelerated by high explosives, has demonstrated its potential to obtain a new type of information from Site $\mathbf{3 0 0}$ hydroshots. ${ }^{23}$ Previously, the velocity of a metal surface was inferred by measuring the distance-time history and differentiating the result with respect to time. Recent success using direct velocity measurements with laser interferometry has shown how much more detailed information can be available, provided that sufficient laser light is reflected by the metal surface. At present we do not know how to consistently prepare the surface such that the return light persists after strong shock loading.

Changes in reflected light can be due to any of several causes. The first is simply an increase in the degree of diffuseness of the target as the shock arrives. This may be closely related to how rough the surface is to start with. A second cause of loss of light can be due to fluff, or small amounts of matter ejected from fine pits and scratches on the surface. A third cause can be a change in the inherent reflectivity, due to changes in structure of the electron-conduction bands of the metal. Any or all of these may contribute during shock loading. 
The electric gun could be used in routine dry runs, measuring the return light to the laser velocimeter under realistic experimental conditions. Before a proposed Site 300 hydroshot involving laser interferometry is fired, we can prepare several samples of the metal of interest and shock them with the electric gun to the same shock pressure as expected in the upcoming hydroshot. Although the electric gun is limited in pulse length, there is sufficient time to learn whether or not fluff will be ejected, ruining reflectivity, or if the light disappears for any reason. Our experience has shown that for jump-off velocities greater than $2.5 \mathrm{~mm} / \mu \mathrm{s}$, the change in return light occurs in a time that is very short compared to the pulse duration that we could generate with the electric gun system.

\section{SHOCK INITIATION OF EXPLOSIVES}

The electric gun offers an attractive means of performing shock initiation experiments. The impact of an electrically-driven flyer plate produces a welldefined stimulus whose intensity and duration can be independently varied. Experiments are low-cost and there is fast turn-around between experiments.

The pressure-time regime accessible in electric gun experiments differs significantly from pressuretime combinations produced by conventional shock initiation experiments. The electrically driven flyers initiate explosives with short-duration high pressure pulses. Figure 8 shows the range of pressure-time

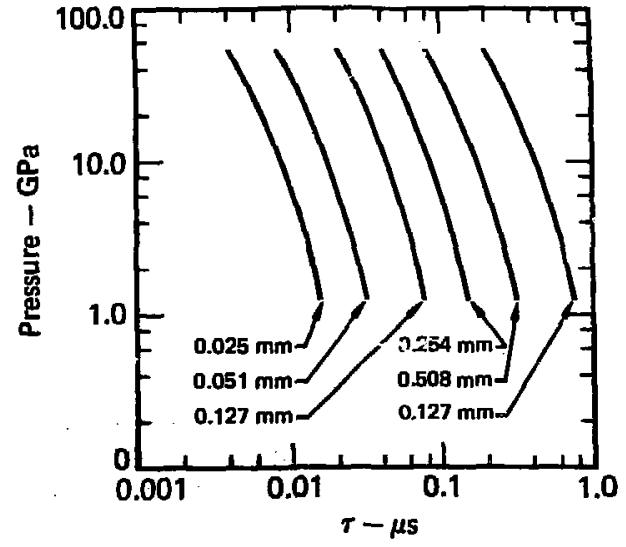

Fig. 8. Range of pressure-time points produced by the slower bank accelerating Kapton Dyers to impact a PBX-9404 target. points produced by our slower bank accelerating Kapton ${ }^{\ominus}$ flyers to impact a PBX-9404 target PBX9404 is a plastic-bonded, HMX-based explosive. For further details, see Ref. 17). Short-pulse initiation experiments will be very useful in developing more realistic theoretical shock initiation models. Our present shock initiation models are fairly successful in predicting the initiation threshold for sustained-pulse initiation experiments, but are not capable of predicting initiation thresholds where very short pulses are employed. The electric gun can provide data to test the capability of improved models.

We have used the electric gun extensively for characterizing the shock sensitivity of various explosives. $^{5,24,25}$ The capability of the electric gun for performing a large number of controlled initiation experiments is unequalled by any other initiation technique. In studying the shock initiation of TATB $(1,3,5-$ triamino-2,4,6-trinitrobenzene), we performed over 900 individual tests in a year. ${ }^{24}$ Figure 9 shows the result of our study of the shock initiation of PBX-9404 by thin flyer plates. Threshold values of pressure versus pulse duration are plotted along with data from earlier short-pulse initiation studies by Gittings ${ }^{26}$ and Trott and Jung. ${ }^{27}$ It should be noted that by using the electric gun we were able to extend the data to significantly higher pressures and shorter pulse widths.

The electric gun provides a better-defined input stimulus than many conventional shock-sensitivity tests, which use explosive charges to drive a shock wave through an inert barrier into the explosive under test (e.g., gap test). The impact of 25-mm-diameter flyer plates accelerated from the electric gun provide truly one-dimensional initiation conditions even for an insensitive explosive like TATB. We feel that a standard shock sensitivity test based on the impact of thin flyer plates accelerated by the electric gun would be a significant improvement over many of the standard tests presently in use. Electric gun experiments have the additional advantage of being applicable to relatively small explosive samples (1-100 g), an important consideration for evaluating new or scarce explosive compositions. To develop such a test we are using the electric gun to perform shock initiation experiments on a number of important explosives, so that we can develop a data base and see how the thin-pulse initiation thresholds correlate with other shock-sensitivity tests.

The electric gun is also capable of probing the two-dimensional aspects of shock initiation in explosives. In our studies of the extremely insensitive 
explosive TATB, $^{24}$ we have observed a dramatic increase in the flyer velocity as the flyer diameter is decreased below $15 \mathrm{~mm}$, as is shown in Fig. 10 .

\section{POSSIBLE DATA SOURCES FOR MAGNETOHYDRODYNAMIC (MHD) CODES}

The foil material that drives the flyer plate in the electric gun is itself an interesting subject for investigation. The foil material is in an extremely-hightemperature, reduced-density state during and after the burst. EOS measurements on the foil material are possible by measuring the acceleration and displacement of the interface between the foil and a transparent buffer material, which acts as a tamper. Such measurements would measure pressure and volume as a function of time. Energy input to the foil could be determined simultaneously by measuring the current and voltage across the foil. Such data would be in a range of interest for imploding liner calculations using MHD codes. The acceleration data could also be compared directly to MHD code calculations, checking the validity of material properties used in the code.

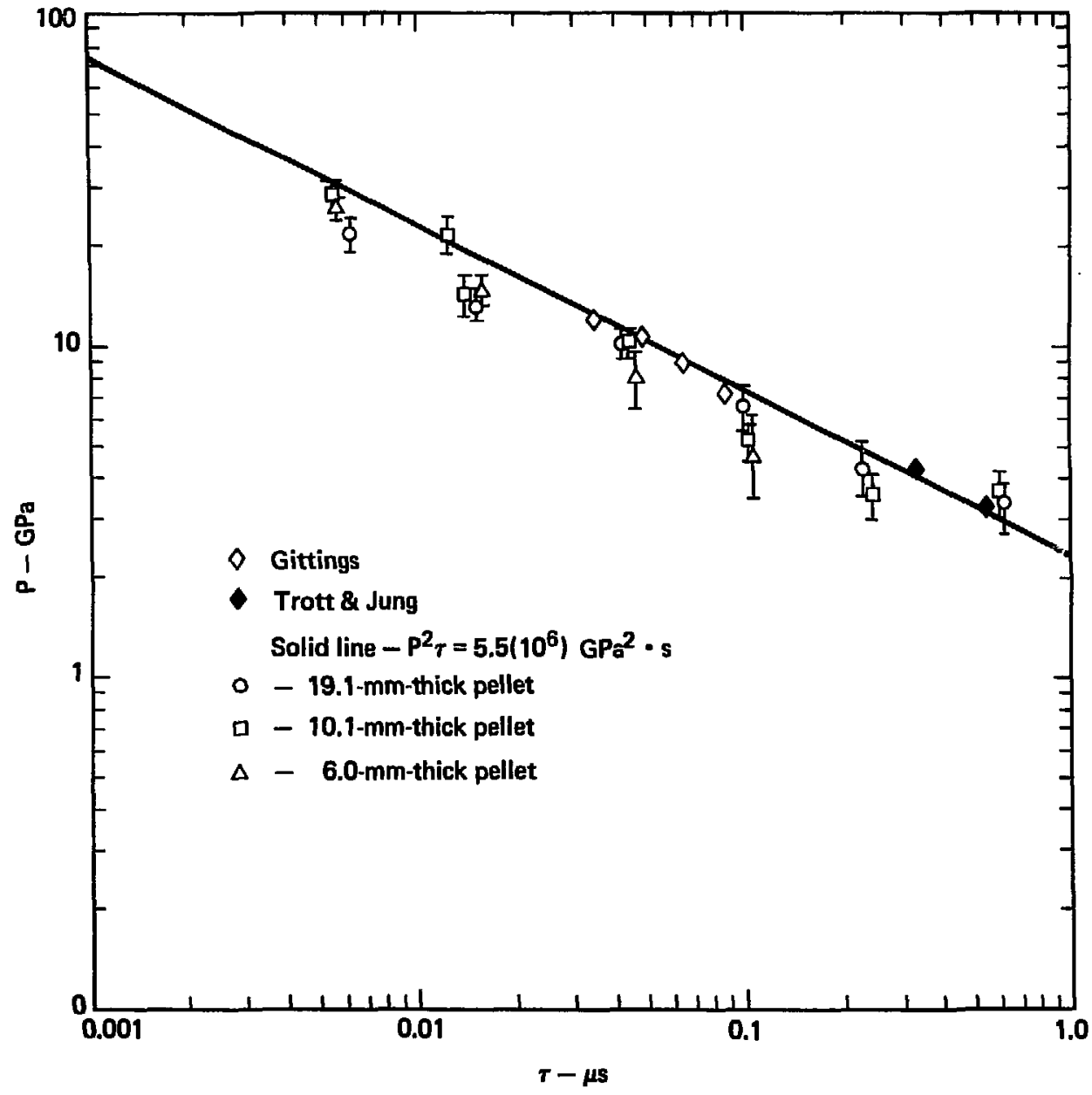

Fig. 9. Shock initiation of PBX-9404 by thin flyer plates. Data are from our own experiments and from those of Gittings ${ }^{26}$ and Trott and Jung ${ }^{27}$. 


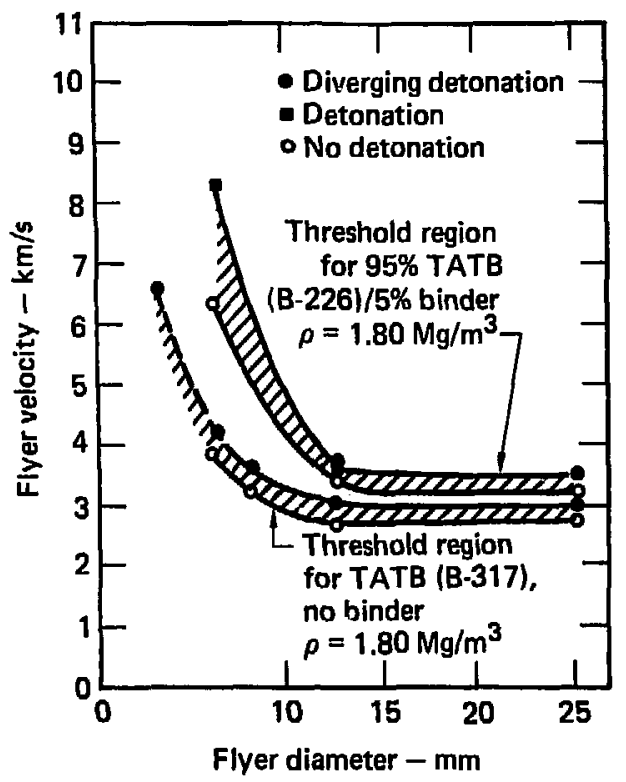

Fig. 10. Flyer velocity threshold regions are shown for two compositions of TATB.

\section{SUMMARY AND CONCLUSIONS}

We have developed a system, the electric gun, that has already been very useful in shock wave studies at LLL and offers unparalleled advantages in many areas of shock wave research. This system has accelerated thin flyer plates to velocities in excess of $20 \mathrm{~km} / \mathrm{s}$ and offers the potential for.precision EOS studies in the 1-t0-5-TPa range.

To date, our greatest use of the electric gun has been in HE shock initiation studies. We have used the electric gun for an intensive study of the explosive TATB 22 and for a survey of the short pulse initiation thresholds of a number of important explosives. 4,23

We recently have begun using the electric gun for high-pressure EOS studies, and have obtained some preliminary data on Hugoniot points for tantalum in the $0.2-\mathrm{TPa}$ range. We feel that the gun is the best candidate as a shock wave generator for EOS studies in the 1-to-S-TPa range, a range that is not accessible using conventional shock wave generators.

An application ideally suited to the unique capa- bilities of the electric gun is the study of the interaction of shock waves with free surfaces. In a thin sample, the electric gun can produce a well-characterized shock wave over an extremely wide range of pressures. The low cost and fast turn-around time permit a large number of experiments relative to other shock wave generators. We have used the electric gun in this capacity in developing laser velocimeters, and we feel that a much greater effort in the surface study area would be very rewarding, particularly with regard to our use of laser diagnostic.

Finally, we wish to point out that our experiments do not yet show that we are approaching any fundamental limit to the flyer velocities achievable with the electric gun. We are confident that we can reach yet higher velocities and pressures with our present bank. We look forward to extending the capability of the electric gun until we can define the physics or engineering constraints that set an upper limit on the flyer velocity. 


\section{ACKNOWLEDGMENTS}

We would like to acknowledge the support and encouragement of G. Staehle and R. Wagner. We are especially appreciative of the hard work and dedication of the Building 345 technicians who assembled and fired the experiments reported here. Finally, we gratefully acknowledge the patience and cooperation of J. Schullerts in preparing the manuscript. 


\section{REFERENCES}

1. D. V. Keller and R. J. Penning, Jr., "Exploding Foils - the Production of Plane Shock Waves and the Acceleration of Thin Plates," in Exploding Wires, W. G. Chase and H. K. Moore, Eds. (Plenum Press, New York, 1962), Vol. 2, p. 263.

2. A. H. Guenther, D. C. Wunsch and T. D. Soapes, "Acceleration of Thin Plates by Exploding Foil Technicues," in Exploding Wires, W. G. Chase and H. K. Moore, Eds. (Plenum Press, New York, 1962), Vol. 2, p. 279.

3. P. W. Duweke, "A Technique for Launching Intermediate Velocity Thin Plastic Sheets," Rev. Sci. Instr. 41, 539 (1970).

4. J. R. Stroud and E. F. Nikkola, Flying Plate ("Slapper") Detonators - Experimental Results, Lawrence Livermore Laboratory, Livermore, CA, UCRL-50938 (1970) (title U, report CRD).

5. R. C. Weingart, R. S. Lee, R. K. Jackson, and N. L. Parker, "Acceleration of Thin Flyers by Exploding Metal Foils: Applications to Initiation Studies," in Proceedings of the Sixth Symposium (International) on Detonation, Office of Naval Research, ACR-221 (1976), p. 653.

6. R. C. Weingart, "Direct Initiation of Acceptor High Explosives," in Military Applications Programs Monthly Report, Lawrence Livermore Laboratory, Livermore, CA, UCRL-50000-69-7, (1969), (title U, report SRD).

7. W. G. Chase and H. K. Moore, Eds., Exploding Wires (Plenum Press, New York, 1969), 4 Vols.

8. F. D. Bennett, "High-Temperature Exploding Wires," in Progress in High Temperature Physics and Chemistry, C. A. Rouse, Ed. (Pergamon, New York, 1968) Vol. 2, p. 1.

9. F. D. Bennett, H. S. Burden, and D. D. Shear, "Expansion of Superheated Metals," J. Appl. Phys. 45, 3429 (1974).

10. T. J. Tucker, "Behavior of Exploding Gold Wires," J. Appl. Phys, 32, 1894 (1961).

11. J, D. Logan, R. S. Lee, R. C. Weingart and K. S. Yee, "Calculation of Heating and Burst Phenomena in Electrically Exploded Foils," J. Appl. Phys. 48, 621 (1971).

12. G. W. Anderson and F. W. Nielson, "Use of the "Action Integral" in Exploding Wire Studies," in Exploding Wires, W. G. Chase and H. K. Moore, Eds. (Plenum Press, New York, 1959), Vol. 1, p. 97.

13. T. J. Tucker and R. P. Toth, Electrical Behavior of Small Exploding Foil Flyer Assemblies, Sandia Laboratories, Albuquerque, NM, SAND-75-0445, (1975).

14. T. J. Tucker and P. L. Stanton, Electrical Gurney Energy: A New Concept of Modeling Energy Transference from Electrically Exploded Conductors, Sandia Laboratories, Albuquerque, NM, SAND-74-0244, (1975).

15. R. W. Gurney, The Initial Velocities of Fragmenis from Bombs, Shells, and Grenades, Battelle Memorial Institute, Columbus, Ohio, Rpt-405, (1943).

16. C. Kittel, Introduction to Solid State Physics (Wiley, New York 1966), p. 78.

17. B. M. Dobratz (Ed), Properties of Chemical Explosives and Explosive Simulants, Lawrence Livermore Laboratory, Livermore, CA, UCRL-52329, Rev. 1, (1974).

18. J. W. Kury, H. C. Hornig, E. L. Lee, J. L. McDonnel, D. L. Ornellas, M. Finger, F. M. Strange, and M. L. Wilkins, "Metal Acceleration by Chemical Explosives," in Proceedings of the Fourth Symposium (International) on Detonation, Office of Naval Research, ACR-126 (1965), p. I.

19. A. E. Siegel, The Theory of High Speed Guns, AGARDograph 91, (North Atlantic Treaty Organization Advisory Group for Aerospace Research and Development, Paris, 1965).

20. C. E. Ragan III, M. G. Silbert, and B. C. Diven, "Shock Compression of Molybdenum to $2.0 \mathrm{TPa}$ by Means of a Nuclear Explosion," J. Appl. Phys. 48, 2860, (1977).

21. L. Veaser and J. Solem, "Studies of Laser-Driven Shock Waves in Aluminum," Phys. Rev. Letters 40, p. 1391, 1978.

22. R. Trainor, H. Graboske, T. Long and J. Shaner, Lawrence Livermore Laboratory, Livermore, CA, Application of High-Power Lasers to Equation-of-State Researcin at Ulirahigh Pressures, UCRL-52562, (1978).

23. H. Chau, D. Goosman, J. Lyle and M. Summers, "A Simple Velocity Interferometer System", Conference on Laser and Electro-Optical Systems, OSA/IEEE, Abstract TUHH4, p. 20 (1978).

24. R. K. Jackson, L. G. Green, R. H. Barlett, W. W. Hofer, P. E. Karmar, R. S. Lee, E. J. Nidik, Jr., L. L. Shaw and R. C. Weingart, "Initiation and Detonation Characteristics of TATB," in Prcceedings of the Sixth Symposium (International) on Detonation, Office of Naval Research, ACR-221 (1976), p. 775. 
25. R. C. Weingart, R. K. Jackson, C. A. Honodel and R. S. Lee, "Shock Initiation of PBX-9404 by ElectricallyDriven Flyer Plates," in Proceedings of the 10th Symposium on Explosives and Pyrotechnics, February, 1979, San Francisco (to be published).

26. E. F. Gittings, "Initiation of a Solid Explosive by a Short-Duration Shock," in Proceedings of the Fourth Symposium (International) on Detonation, Office of Naval Research, ACR-184 (1970), p. 191.

27. 'B. D. Trott and R. G. Jung, "Effect of Pulse Duration on the Impact Sensitivity of Explosives," in Proceedings of the Fifth Symposium (Intemational) on Detonation, Office of Naval Research, ACR-184 (1970), p. 191. 